\title{
Gravitational Field Propulsion
}

\author{
Walter Dröscher ${ }^{1}$, Jochem Hauser ${ }^{2 *}$ \\ ${ }^{1}$ Institut für Grenzgebiete der Wissenschaft, 6010 Innsbruck, Austria \\ ${ }^{2}$ Faculty Karl-Scharfenberg, Univ. of Applied Sciences, Salzgitter Campus, 38229 Salzgitter, Germany
}

Current space transportation systems are based on the principle of momentum conservation of classical physics. Therefore, all space vehicles need some kind of fuel for operation. The basic physics underlying this propulsion principle severely limits the specific impulse and/or available thrust. Launch capabilities from the surface of the Earth require huge amounts of fuel. Hence, space flight, as envisaged by von Braun in the early 50s of the last century, will not be possible using this concept. Only if novel physical principles are found can these limits be overcome. Gravitational field propulsion is based on the generation of gravitational fields by man made devices. In other words, gravity fields should be experimentally controllable. At present, it is believed that there are four fundamental interactions in physics: strong (nuclei), weak (radioactive decay), electromagnetic and gravitational. As experience has shown for the last six decades, none of these physical interactions is suitable as a basis for novel space propulsion. None of the advanced physical theories, like string theory or quantum gravity, go beyond the four known interactions. On the contrary, recent results from causal dynamical triangulation simulations indicate that wormholes in spacetime do not seem to exist, and thus even this type of exotic space travel may well be impossible. However, recently, novel physical concepts were presented that might lead to advanced space propulsion technology, based on two novel fundamental force fields. These forces are represented by two additional long range gravitational-like force fields that would be both attractive and repulsive, resulting from interaction of gravity with electromagnetism. A propulsion technology, based on these novel long range fields, would be working without propellant. The current theoretical and experimental concepts pertaining to the novel physics of these gravity-like fields are discussed together with recent gravitomagnetic experiments performed at ARC Seibersdorf (2008). The theoretical concepts of Extended Heim Theory, EHT, are employed for the explanation of these experiments.

\footnotetext{
${ }^{* 1}$ Senior Scientist, Institut für Grenzgebiete der Wissenschaft, 6010 Innsbruck, Austria

$* 2$ Prof., Faculty Karl-Scharfenberg, Univ. of Applied Sciences, 38229 Salzgitter, Senior member AIAA.
} 


\section{Experimental and Theoretical Concepts of Novel Field Propulsion}

$\mathrm{T}$ HE current status of space propulsion is characterized by two contradicting scenarios. The first one, chemical propulsion delivers high thrust but for several minutes only at relatively low specific impulse, and is used today to lift heavy payloads from the surface of the Earth into nearby space (for instance LEO). The second one, electric and plasmadynamic propulsion, provides low thrust over longer periods of time (up to several months) at high specific impulse, and is employed in scientific interplanetary missions of long duration. Propulsion systems can be classified according to their physical principles as thermal propulsion systems or electromagnetic propulsion systems. Advanced versions of these systems are described in the recent book by Bruno et al., ${ }^{17}$ which performs a linear extrapolation of present technology, envisaged to be realizable in 2020. Another class of advanced concepts using photonic propulsion, solar sails, or laser propulsion has been suggested. Comparing these advanced concepts with the space propulsion concepts discussed in the books by Seifert et al. (1959) ${ }^{1}$ and Corliss $(1960)^{2}$ it becomes obvious that the physical principles of all of these concepts have been around for several decades, but with regard to performance no significant progress has been achieved. For instance, electric propulsion systems were already tested in the 1960s and so was nuclear propulsion. Chemical propulsion systems were never more powerful than in the 1960s.

The reason for this lack in progress is that physical laws pose strict limits on the practicality and the performance of even the most advanced propulsion systems and in practice have prevented the construction of efficient and effective propulsion systems. First, all systems considered so far operate on the basis of expulsion of mass and energy, i.e., have to obey classical momentum conservation. Hence, some kind of propellant needs to be provided. Second, the speed of light in vacuum is limited by special relativity, so interstellar travel in general does not seem to be feasible in our spacetime. This, however, is not at all a concern at present, since our current chemical propulsion systems are delivering velocities of about $10 \mathrm{~km} / \mathrm{s}$.

The state of the art of different types of advanced space propulsion concepts, based on more sophisticated physics, like space drives, warp drives, or gravity control are described in Davis and Millis (eds.) ${ }^{3}$. Nevertheless, these concepts are all utilizing one of the known four fundamental physical interactions. For instance, they are making use of special properties of the spacetime metric of general relativity $(G R)$, or try to exploit quantum entanglement for faster than light travel. Although these concepts have been known, too, in physics since the late 1930s, their engineering realization seems to be as unlikely today as it was at the time of their discovery. In particular, faster than light approaches in general relativity, $G R$, as investigated by Davis, Chapter $15, \mathrm{in}^{3}$ probably are ruled out by novel causal dynamical triangulation computer simulations ${ }^{4-6}$, since realistic spacetime topologies do not seem to allow this kind of traversable wormholes, and this kind of interstellar travel might be unfeasible.

On the other hand, current physics has no explanation for the existence of exactly four fundamental forces that is, there is a belief only on the existence of four fundamental interactions ${ }^{7,8}$. The question therefore arises, are there any additional fundamental physical interactions? Perhaps it is classical physics and not quantum mechanics that is incomplete that is, there might exist additional long range interactions. This question was already discussed in detail in several recent papers, for instance ${ }^{9,12-14}$. Since 2002, novel physical ideas have been presented under the name Extended Heim Theory, $(E H T)$, ${ }^{1}$ postulating the existence of six fundamental forces. According to $E H T$, there should be three gravitational forces in combination with the known electromagnetic, weak, and strong forces. Beside Newtonian gravitation (graviton, $v_{g}$, attractive), EHT requires the existence of two additional gravitational fields, termed gravitophoton interaction, $v_{g p}^{0}$ (both attractive and repulsive), which results from the conversion of electromagnetic energy into gravitational energy, and quintessence, $v_{q}$, (repulsive) ${ }^{9,12}$. The geometric approach, namely describing physical interactions by metric tensors and the underlying physical concepts of $E H T$ are briefly presented in Sec. II.

The question naturally arises about the physical relevance of theses ideas. Are there any, hitherto unknown, physical phenomena that might justify the existence of additional physical interactions? The answer seems to be affirmative. In March 2006, the European Space Agency (ESA), on their webpage, announced credible experimental results, reporting on the generation of both gravitomagnetic (termed frame dragging in $G R$, which, however, is too small to be measured in a laboratory on Earth) and gravity-like or gravitoelectric fields, which are acceleration fields $b$ performed at ARC Seibersdorf, Austria. Since then further experimental results have been published by Tajmar et al. from ARC ${ }^{29-31}$ and, in July 2007, Graham et al. published a paper on the generation of a gravitomagnetic field

\footnotetext{
${ }^{\text {a }}$ It should be noted thatEHT does not have reached the status of physical theory. At present, it is a classification scheme to construct a polymetric tensor that possibly encompasses all physical interactions ${ }^{9,12,13}$ and is an approach to geometrize physics as envisaged by Einstein ${ }^{16}$ as well as $\mathrm{Heim}^{18}$ in 1952 and Finzi $^{27}$ in 1955.

${ }^{\mathrm{b}}$ In analogy to electromagnetism, gravity-like fields are denoted as gravitoelectric fields $\mathbf{E}_{G}$ since they actually produce an acceleration. One speaks of a gravitoelectric force if the $\mathbf{E}_{G}$ field is generated by a stationary mass. The term gravitomagnetic force is used if $\mathbf{E}_{G}=\mathbf{v} \times \mathbf{B}_{G}$, i.e. produced by a rotating mass together with a mass density current.
} 


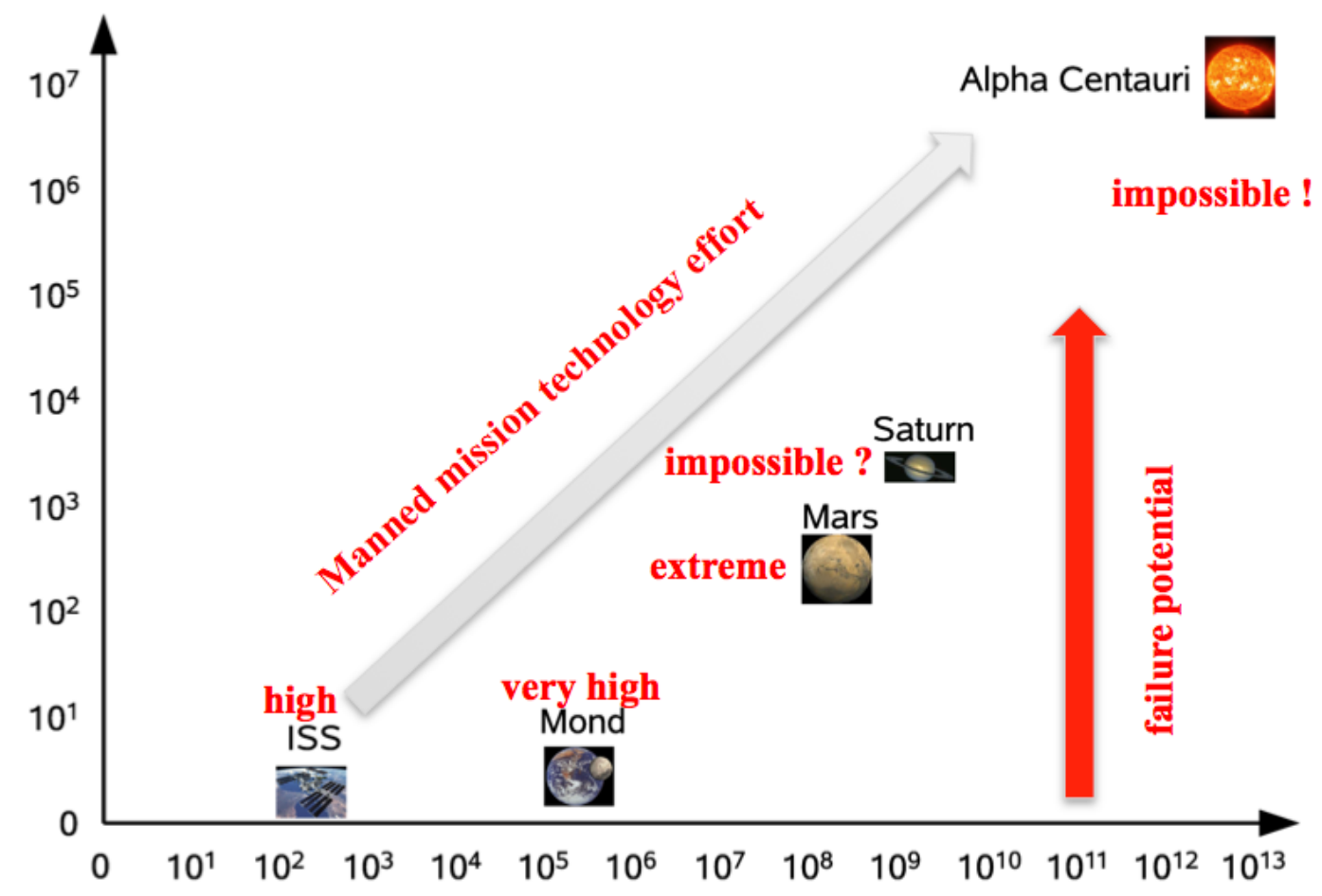

Figure 1. The picture shows the rapidly increasing mission difficulty and hazard with flight distance. The abscissa depicts distance in $\mathbf{~ k m}$ while the ordinate shows the travel time in days. Space flight as envisaged by von Braun cannot be achieved within the stringent limits posed by the currently four known fundamental physical interactions.

produced by a cryogenic lead disk, but using a completely different measurement technique ${ }^{32}$, see Table 4 . However, their results are not conclusive, since the sensitivity of their ring laser was about two orders of magnitude lower than the gyro employed at ARC. In addition, in 2008 Tajmar et al. ${ }^{33}$ published a more comprehensive set of gravitomagnetic experiments. In Sec. III] EHT will be used to present a qualitative explanation for these results. Furthermore, in 2007 results of the NASA Stanford Gravity-Probe B (GP-B) experiment ${ }^{15}$ became available, and $E H T$ was used to model the gyro anomaly seen in this experiment as well as the acceleration and deceleration of the two gyro pairs ${ }^{9}$.

Naturally, such a propellantless propulsion system would be far superior over any existing propulsion technology, while its technology might be substantially simpler and cleaner than chemical, fission, or fusion rockets. There is, of course, insufficient knowledge at present, both theoretical and experimental, to guarantee the realization of such a device. However, the benefits of such a device are formidable.

\section{Physical Concepts of EHT for Gravitational Field Propulsion}

The physical concepts of $E H T$ were laid out in previous publications, see for instance ${ }^{9,12-14}$. The interpretation of the physical equations for the postulated gravitophoton field leads to the conclusion that this field could be used to accelerate a material body without the use of propellant by generating a vertical gravitational field. Therefore, gravitation, as we know it seems to be composite, and according to EHT comprises three interactions, mediated by the graviton $\left(v_{g}\right.$, attractive), gravitophoton $\left(v_{g p}^{0}\right.$, attractive and repulsive), and the quintessence or vacuum $\left(v_{q}\right.$, repulsive) particle that is, there there should be three field quanta of gravitation. This means that the gravitational constant $G$ contains contributions of all three gravitational constants, termed $G_{g}, G_{g p}$ and $G_{q}$, respectively.

\section{A. The Nature of Gravity}

In $E H T$ two additional gravity-like fields occur, and therefore the set of three coupled fields needs to be considered. Moreover, a conversion of photons, $\gamma$ into gravitophotons, $v_{g p}^{0}$, can take place, coupling electromagnetism with gravitation, which leads to the generation of strong gravitomagnetic fields in comparison with GR. Hence, classical physics as conceived by $\boldsymbol{E H T}$ may, under special experimental conditions, lead to hitherto unknown phase transitions, and 
thus exhibit completely novel physical phenomena.

Because of the coupling of electromagnetism with gravitation, the complete set of of equations should be considered, described of course by the Einstein field equations, which are the structural equations for all interactions. However, for the time the set of gravitational equations is considered only.

- In classical $G R$, the Lense-Thirring frame dragging effect exists, as was confirmed by GP-B, and thus the graviton, $v_{g}$, is capable of generating both the gravitoelectric, $\mathbf{E}_{g}$, as well as the gravitomagnetic, $\mathbf{B}_{g}$ field. In $G R$ it is these fields only that appear in the Einstein field equations.

- The quintessence quantum $v_{q}$, as the dual exchange particle to the graviton, also should have the capability of producing a gravitomagnetic field, termed $\mathbf{B}_{q}$, although the interaction strength is assumed to be much smaller.

- In the gravitomagnetic experiments by Tajmar et al. (no acceleration field) a much larger gravitomagnetic field than predicted by $G R$ was measured, which is denoted as $\mathbf{B}_{g p}$. Obviously, because of its magnitude, this field is not generated by gravitons, and thus is not described by the field $\mathbf{B}_{g}$. Consequently, the quanta of this field are not gravitons, $v_{g}$, but the above mentioned gravitophotons, $v_{g p}^{0}$. Instead, the gravitophoton, $v_{g p}^{0}$, is deemed to be responsible for its existence. The gravitophoton is believed to emerge from photon conversion, i.e., from the mechanism of converting photons into gravitophotons, which eventually leads to the strong gravitomagnetic field observed.

Therefore the total gravitomagnetic field actually measured is

$$
\mathbf{B}_{G}:=\mathbf{B}_{g}+\mathbf{B}_{g p}+\mathbf{B}_{q},
$$

and thus should be a composite field. Clearly, it is depending on the experimental situation which of the fields is the dominating one. In the well known situation of rotating celestial bodies, only the $\mathbf{B}_{g}$ seems to occur. In case a phase transition occurs, in the experiments by Tajmar et al., Graham et al. and possible in the GP-B experiment, ${ }^{9}$ triggered by cryogenic temperature, the much larger $\mathbf{B}_{g p}$ seems to appear. In case quintessence quanta are generated, the gravitomagnetic quintessence field $\mathbf{B}_{q}$ should be observed. It is assumed that the $\mathbf{B}_{q}$ field is much weaker, because of the smaller coupling constant $G_{q}$ of the repulsive quintessence force. It should be noted that only the sum of the divergence of the three fields needs to be zero, but not for the individual fields.

It is well known that the linearized Einstein equations have a structure similar to the Maxwell equations of electrodynamics. In $E H T$, these linearized equations are to be satisfied by the total gravitoelectric $\mathbf{E}_{G}$ and gravitomagnetic $\mathbf{B}_{G}$ fields.

$$
\nabla \cdot \mathbf{B}_{G}=\nabla \cdot \mathbf{B}_{g}+\nabla \cdot \mathbf{B}_{g p}+\nabla \cdot \mathbf{B}_{q}=0
$$

This means that a coupling between the three fields can exist. The source of one field might become the sink of another field and mixing among the fields might occur. The mathematical implications have not yet been fully investigated.

With regard to gravitoelectric fields the situation seems to be somewhat different in that the total gravitoelectric field $\mathbf{E}_{G}=\mathbf{E}_{g}+\mathbf{E}_{q}$ or $\mathbf{E}_{G}=\mathbf{E}_{g p}^{+}+\mathbf{E}_{g p}^{-}$are depending on the two decay channels of the neutral gravitophoton $v_{g p}^{0}$ as depicted in Fig 3 .

For a qualitative explanation of Tajmar's observations, the following, somewhat hand-waving, arguments might be considered. Could it be that in the experiments by Tajmar et al. factors $c$ and/or $G$ undergo drastic change, caused by the proposed interaction between electromagnetism and gravitation, manifesting itself as phase transition ? This process would be similar to electrodynamic phenomena in matter. If and how Maxwell's equations are coupled to the linearized Einstein equations, as they might, if there is an interaction between the two forces, has not been established so far.

If spacetime is made of discrete pieces that is, atoms of spacetime exist, e.g. ${ }^{11}$, then spacetime might be susceptible to collective modes, representing a daunting many-body problem. A major rearrangement of the many-atom spacetime ground state could take place in the new symmetry-broken phase. Each phase of spacetime, similar to phenomena observed in condensed matter physics, may exhibit its proper fundamental symmetry, characterizing this phase. Hence, spacetime would assume the role of physical field(s) (particles), and therefore should be accounted for in all physical processes of conservation of energy and momentum. These remarks should only serve as general qualitative explanation for the recently observed large gravitomagnetic effects.

Fig. 5 depicts the experiments of Tajmar et al., where a cryogenic $\mathrm{Nb}$ ring is subjected to angular acceleration, which should lead to a gravitophoton force. EHT makes the following predictions for the measured gravitational fields that are attributed to photon-gravitophoton interaction. 
- For the actual experiment, shown in Fig. 5(Tajmar et al.), the gravitophoton force is in the azimuthal direction, caused by the angular acceleration of the superconducting niobium disk. The acceleration field is opposite to the angular acceleration, obeying some kind of Lenz rule.

- For the novel experiment of Fig. 6(field propulsion experiment), a force component in the axial direction should be generated.

\section{B. Building Blocks of Physics}

In order for physical events to manifest themselves in our four-dimensional spacetime three basic building blocks have to be present. The first one is the existence of four-dimensional spacetime, termed also external spacetime, that acts as the stage on which all physical events occur. The second building block is an internal space, called Heim space, denoted $\mathrm{H}^{8}$, which is responsible for the existence of the physical actors, namely the physical interactions and matter. Each of th interactions or material particles is described by its so called Hermetry form (hermeneutics of geometry, i.e., in the present case physical meaning of geometry). A Hermetry form is a special metric tensor resulting from the double coordinate system transformation mandated by the existence of internal Heim space ${ }^{12,14}$ ), see Sec. D The third building block is the substructure or subgroup structure that each Hermetry form possesses, since it metric comprises a set of partial terms. The subgroup describes, for instance, the number of different particles in the group.

\section{Six Fundamental Physical Forces}

The poly-metric tensor constructed in $E H T$ gives rise to six fundamental forces (interactions) that are depicted in Fig. 2. Since $G R$ uses pure spacetime only, as a consequence, there is only one metric tensor and hence only part of the physical world is visible in the form of Newtonian gravitation. In order to describe all physical forces, the poly-metric tensor resulting from Heim space needs to be employed, see for instance ${ }^{14}$.

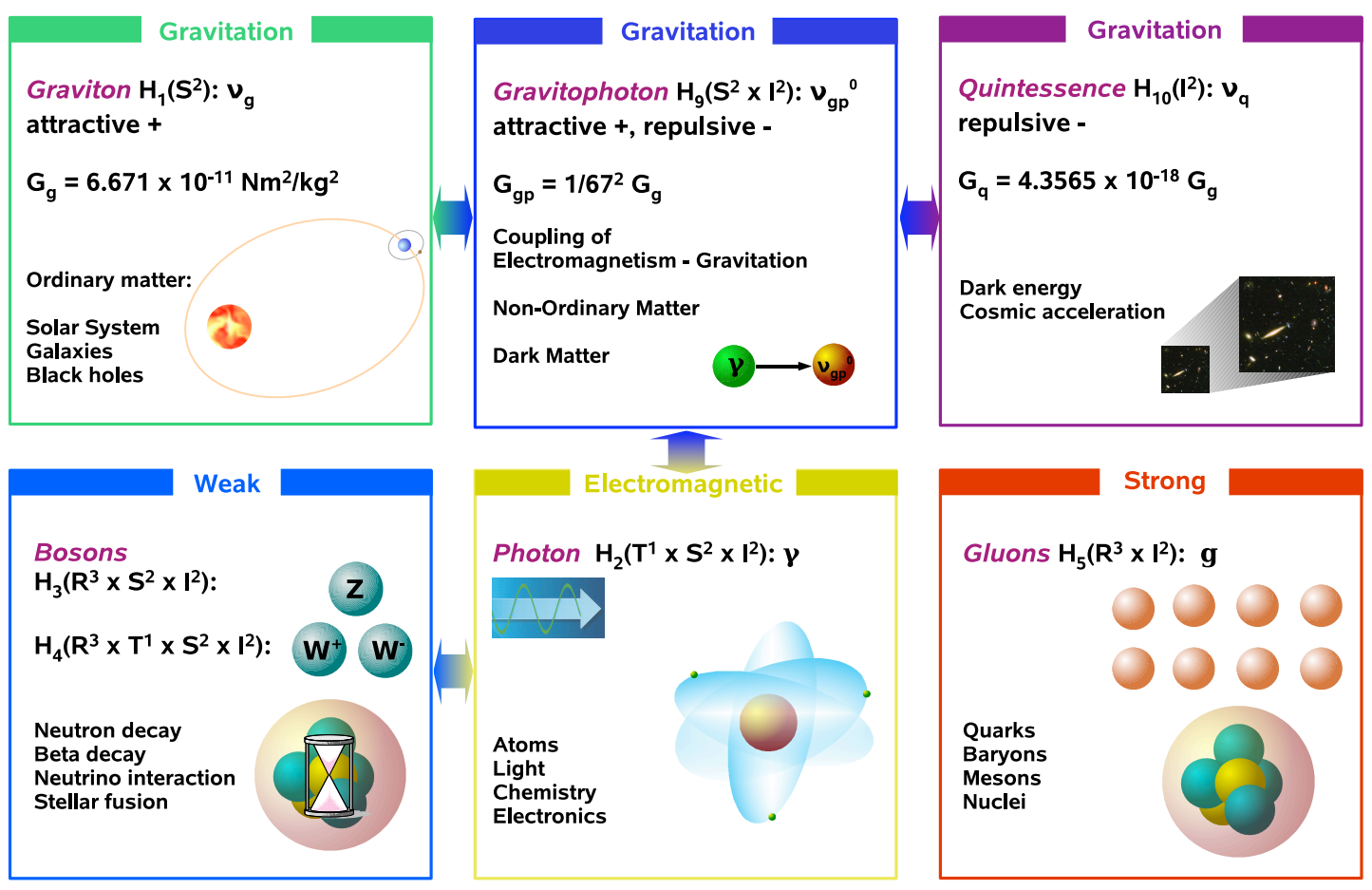

Figure 2. Six fundamental forces are predicted by $E H T$. Three of them are gravity-like fields (upper row, coupling strengths), mediated by three field quanta termed graviton (attractive), gravitophoton (attractive and repulsive), and quintesssence particle (repulsive). The second row shows the electromagnetic, weak, and strong interactions. Arrows indicate possible coupling between interactions. Corresponding Hermetry (metric tensors) forms are listed in Tables 1 and 2.

This idea was first conceived by the German physicist B. Heim. A similar principle was mentioned by the Italian mathematician B. Finzi. The poly-metric tensor of $E H T$, resulting from the concept of $\mathrm{H}^{8}$ internal symmetry space and its four subspaces, is subdivided into a set of metric sub-tensors. Each element of this set, denoted as Hermetry form, 
is equivalent to a physical interaction (e.g. gluons, see Table 1) or class of particles (e.g. charged leptons, see Table 11, and thus the geometrization of physics may be achieved. Of course, the question remains how to construct the energy-momentum tensor from the metric tensor in order to close the system of equations. An open question still is the mass spectrum of elementary particles, which should be obtained from an eigenvalue equation and also the lifetimes of particles. This is, in a nutshell, the strategy chosen, based on the ideas of Heim and Finzi, to accomplish Einstein's goal of the geometrization of physics $\mathrm{C}$

It must be noted that this approach is in stark contrast to elementary particle physics, in which particles possess an existence of their own, and spacetime is just a background staffage ${ }^{36}$. In $E H T$, considered as the natural extension of $G R$, matter seems to be a consequence of the internal space $\mathrm{H}^{8}$. These two physical pictures are mutually exclusive, and experiment will show which view ultimately reflects physical reality. It is, however, well understood that the concept of a pointlike elementary particle is highly useful as a working hypothesis in particle physics.

\section{Hermetry Forms: Ordinary and Non-Ordinary Matter}

Naturally, the number and type of interactions depend on the structure of internal space $\mathrm{H}^{8}$ whose subspace composition along with the physical meaning of the individual subspaces was discussed in ${ }^{9,12-14}$. Contrary to the ideas employed in String theory, see for example ${ }^{37}, \mathrm{H}^{8}$ is an internal space of 8 dimensions comprising four subspaces denoted $\mathbb{R}^{3}, \mathrm{~T}^{1}, \mathrm{~S}^{2}, \mathrm{I}^{2}$.

In mathematical terms, $H^{8}$ is the direct sum of four subspaces, i.e., $H^{8}=\mathbb{R}^{3} \oplus \mathrm{T}^{1} \oplus \mathrm{S}^{2} \oplus \mathrm{I}^{2}$. This means that $\operatorname{dim} \mathrm{H}^{8}=\operatorname{dim} \mathbb{R}^{3}+\operatorname{dim} \mathrm{T}^{1}+\operatorname{dim} \mathrm{S}^{2}+\operatorname{dim} \mathrm{I}^{2}=3+2+2+1$.

Furthermore, the decomposition for any vector $|a\rangle \in \mathrm{H}^{8}$ is unique. With the introduction of the four subspaces of $\mathrm{H}^{8}$ a symmetry breaking has been introduced, which is causing the formation of physical entities as well as physical structures via Hermetry forms, see below.

In physical terms, the $\mathbb{R}^{3}$ coordinates are responsible for the existence of mass, $T^{1}$ coordinate for the existence of charges, $S^{2}$ for the formation of organizational structures, and $I^{2}$ for information structures.

This symmetry breaking has been introduced ad hoc, to account for the fact that the Universe in its evolution has the possibility to develop massive particles as well as charges. This is, however, entirely justified by observations.

$\mathrm{H}^{8}$ is supposed to be a vector space over the field of quaternions $\mathbb{H}$. The elements of $\mathrm{H}^{8}$ are vectors of quaternions, their dimension being equal to one of the four subspaces. The internal coordinates $\xi^{a}$ with $a=1, \ldots, 8$ are the real part of the quaternions. Quaternions $q=a+i b+c j+d k\left(q q^{*} \in \mathbb{R}\right)$ are an extension of the complex numbers $c=a+i b$ by defining two additional imaginary units $j$ and $k$, where, however, $k:=i j, i^{2}=-1, j^{2}=-1, i j+j i=0$. The algebra $\mathbb{H}$ is the simplest non-commutative algebra possible.

To each Hermetry form, whose metric tensor is composed from the coordinates of the four subspaces, its proper symmetry group is associated, leading to a hierarchical group structure. That is, there seems to be no single monster group comprising all conceivable physics. In turn, each Hermetry form comprises its own specific set of partial metric terms. So far the correspondence between these terms and their symmetry group has not been worked out.

For instance, the graviton, $v_{g}$, Hermetry form $H_{1}$, is described by the group of spacetime symmetries (Lorentz and Poincaré) $S O(3,1)$ and $P(3,1)$. The photon, $\gamma$, denoted by $H_{2}$, has symmetry $U(1)$ etc., for the complete representation see Table 1

However, there is also the table of non-ordinary matter whose Hermetry forms lead to novel groups, such as for the neutral gravitophoton, $v_{g p}^{0}$ denoted by Hermetry form $H_{9}$ and represented by symmetry group $S O(4)$, see Table 2 Hence, $\mathrm{H}^{8}$ allows the construction of a poly-metric, while in string theory only a higher-dimensional mono-metric exists. Although this mono-metric tensor can be further subdivided (broken symmetry) in order to give the four known physical forces, its ad-hoc construction does not provide the stringent fundamental physical insights from which the complete set of physical interactions can be derived.

The two matter tables depict the classification scheme for physical interactions and particles as obtained from the poly-metric of space $\mathrm{H}^{8}$ or Heim space. Superscripts for subspaces indicate dimension. A Hermetry form characterizes either a physical interaction or class of particles, and is represented by the metric of an admissible subspace (a space thus has real physical meaning) of $\mathrm{H}^{8}$, which is a combination of the four elementary subspaces as mentioned above. Any admissible subspace combination needs $\mathrm{S}^{2}$ or $\mathrm{I}^{2}$ coordinates to be present in order to realize physical events in our spacetime. The only exception is the Hermetry form $H_{16}$ for the Higgs field. Employing this selection rule leads to 12 admissible Hermetry forms, plus three so called degenerated Hermetry forms, and together with the special Hermetry form of the Higgs field (subspace $\mathbb{R}^{3}$ only) there exists a total of 16 Hermetry forms. The four different colors in the messenger particle column indicate the four known fundamental interactions. Any Hermetry form containing subspace

${ }^{\mathrm{c}}$ There is of course a further aspect, namely the quantization of the associated metric fields that should result in the respective mediator bosons. 
$\mathbb{R}^{3}$ is associated with ordinary (real matter), see Tables 1 . 3 . Although gluons are supposed to have zero mass, the mass of the proton, about $1 \mathrm{GeV}$, is much larger than the sum of the masses of its three quarks, uud, which amount to some $10 \mathrm{MeV}$. Within the proton radius the interaction energy between the three quarks, as permeated by the gluons, i.e. their color fields, contributes the missing mass. Therefore, it is reasonable to assume that subspace $\mathbb{R}^{3}$ occurs in the Hermetry form for gluons, $H_{5}$. Moreover, the presence of $\mathbb{R}^{3}$ in the neutrino Hermetry form $H_{7}$ requires that neutrinos have real mass. Furthermore, the combination of subspaces $\mathbb{R}^{3}$ and $\mathrm{T}^{1}$ indicates charged particles of real mass. The correspondences between Hermetry forms of Tables 1 and 2 should be noted, in particular the correspondence between neutrinos and dark matter.

\begin{tabular}{|l|l|l|l|}
\hline \multicolumn{4}{|c|}{ Hermetry Forms of Ordinary Matter (OM) } \\
\hline $\begin{array}{c}\text { Hermetry form } \\
\text { Lagrange density }\end{array}$ & $\begin{array}{c}\text { Messenger } \\
\text { particle }\end{array}$ & $\begin{array}{c}\text { Symmetry } \\
\text { group }\end{array}$ & $\begin{array}{l}\text { Physical } \\
\text { interaction }\end{array}$ \\
\hline$H_{1}\left(S^{2}\right), L_{g}$ & $\nu_{g}$ graviton & $S O(3,1)$ & gravitation + \\
\hline$H_{2}\left(T^{1} \times S^{2} \times I^{2}\right), L_{e m}$ & $\gamma$ photon & $\mathrm{U}(1)$ & $\begin{array}{l}\text { electromagnetic } \\
\gamma \rightarrow \nu_{g p}^{0} \\
\text { only the photon } \\
\text { interacts with } N O M\end{array}$ \\
\hline$H_{3}\left(R^{3} \times S^{2} \times I^{2}\right), L_{w e}$ & $Z^{0}$ boson & $\mathrm{SU}(2)$ & weak \\
\hline$H_{4}\left(R^{3} \times T^{1} \times S^{2} \times I^{2}\right)$ & $W^{ \pm}$bosons & $\mathrm{SU}(2)$ & weak \\
\hline$H_{5}\left(R^{3} \times I^{2}\right), L_{s}$ & $g$ gluons & $S U(3)$ & strong \\
\hline$H_{6}\left(R^{3} \times T^{1} \times S^{2}\right)$ & $e, \mu, \tau$ & & charged leptons \\
\hline$H_{7}\left(R^{3} \times S^{2}\right)$ & $\begin{array}{l}\nu_{e}, \nu_{\mu}, \nu_{\tau} \\
\text { neutrinos }\end{array}$ & & neutral leptons \\
\hline$H_{8}\left(R^{3} \times T^{1} \times I^{2}\right)$ & $q$ & & quarks \\
\hline \hline
\end{tabular}

Table 1. Table of Hermetry forms for ordinary matter $(O M)$ describing all messenger particles (gauge bosons), namely graviton, photon, vector bosons, and gluons as well as all known types of matter (last three blue rows), i.e., leptons and quarks. The gauge bosons comprise the four known fundamental forces. However, these forces are not sufficient to explain the experiments by Tajmar et al. and Graham et al., as was shown in' nor can they account for dark matter or dark energy.

The two additional gravitational fundamental forces are mediated by gravitophotons (attractive, $v_{g p}^{+}$and repulsive, $v_{g p}^{-}$) as well as the quintessence particle (repulsive, $v_{q}$, dark energy). The quintessence particle, $v_{q}$, is assumed to be responsible for the interaction between spacetime (vacuum field) and ordinary matter.

Any current of imaginary electrons or positrons can generate an imaginary vector potential, $\mathbf{A}_{I}$, that can interact with imaginary charges to produce a real physical effect. These particles will eventually disappear, because of strict charge conservation, and the resulting electromagnetic interaction is converted into a gravitational field. This phenomenon might be the cause for the observed extremely strong gravitomagnetic and gravity-like fields in the experiments by Tajmar et al.

The concept of imaginary matter in Table 2 should not be taken as if there existed a new type of matter, since these particles are assumed to be virtual particles of imaginary mass that is, they do not occur in the initial and final states of a reaction.

In EHT, dark matter is composed of a new class of particles, the NOM neutral leptons (fermions), but these are not WIMPS (Weakly Interacting Massive Particles) whose masses are supposed to be hundreds of GeV, and thus have elucidated present accelerators. The inertial masses of $e^{0}, \mu^{0}, \tau^{0}$ have not been calculated, but are assumed to be close to their charged counterparts, i.e., $0.511 \mathrm{MeV} / \mathrm{c}^{2}$ for electrons, $105.66 \mathrm{MeV} / \mathrm{c}^{2}$, and $1.78 \mathrm{GeV} / \mathrm{c}^{2}$ (compared to 938 $\mathrm{MeV} / \mathrm{c}^{2}$ for protons).

If $e^{0}, \mu^{0}, \tau^{0}$ existed in Nature, the question naturally arises: why did not accelerators already long ago produce these particles? Accelerators or colliders produce beams of high-energy electrons or protons that are driven onto a target, or two beams are colliding from opposite directions. According to Table 1 and also in accordance with the Standard Model, there is no place for $O M$ neutral leptons, except for the almost massless neutrinos, which cannot contribute more than $1 \%$ to dark matter. In $E H T$, however, the $N O M$ counterpart to neutrinos, as can be seen from 


\begin{tabular}{|l|l|l|l|}
\hline \multicolumn{1}{|c|}{ Hermetry $\begin{array}{c}\text { Fermetry form } \\
\text { Lagrange density }\end{array}$} & $\begin{array}{c}\text { Messenger } \\
\text { particle }\end{array}$ & $\begin{array}{c}\text { Symmetry } \\
\text { group }\end{array}$ & $\begin{array}{c}\text { Physical } \\
\text { interaction }\end{array}$ \\
\hline$H_{1}\left(S^{2}\right), L_{g}$ & $\nu_{g}$ graviton \\
$H_{9}\left(S^{2} \times I^{2}\right), L_{g p}$ & $\begin{array}{l}\nu_{g p}^{0} \rightarrow\left\{\begin{array}{l}\nu_{g}+\nu_{q} \\
\nu_{g p}^{+}+\nu_{g p}^{-} \\
\text {gravitophotons } \\
\nu_{q} \text { quintessence } \\
\text { particle }\end{array}\right. \\
H_{10}\left(I^{2}\right), L_{q}\end{array}$ & $S O(4)$ & gravitation + \\
\hline$H_{11}\left(T^{1} \times S^{2} \times I^{2}\right)^{*}$ & $\gamma_{I}$ & gravitation \pm \\
\hline$H_{12}\left(I^{2}\right)^{*}$ & $g_{I}$ & $U(1)$ & $\begin{array}{l}\text { gravitation }- \\
\text { dark energy }\end{array}$ \\
\hline$H_{13}\left(T^{1} \times S^{2}\right)$ & $e_{I}^{-}, e_{I}^{+}$ & $\begin{array}{l}\text { interaction bosons } \\
\text { for charged } \\
\text { Im-leptons }\end{array}$ \\
\hline$H_{14}\left(R^{3} \times S^{2}\right)^{*}$ & $\begin{array}{l}e^{0}, \mu^{0}, \tau^{0} \\
\text { dark matter }\end{array}$ & $\begin{array}{l}\text { interaction bosons } \\
\text { for Im-quarks }\end{array}$ \\
\hline$H_{15}\left(T^{1} \times I^{2}\right)$ & $q_{I}$ & $\begin{array}{l}\text { charged } \\
\text { Im-leptons }\end{array}$ \\
\hline \hline
\end{tabular}

Table 2. Table of Hermetry forms for non-ordinary matter (NOM). First, it should be noted that this table contains imaginary matter in the form of imaginary electrons and positrons (imaginary mass, but real charge), which, however, are virtual particles, denoted as $e_{I}^{ \pm}$together with its messenger particle $\gamma_{I}$. Second, the existence of neutral leptons is postulated.

\begin{tabular}{|l|l|l|l|}
\hline \hline$H_{16}\left(R^{3}\right)$ & $\phi=\frac{1}{\sqrt{2}}\left(\phi_{1}+i \phi_{2}\right)$ & spacetime & Higgs field \\
\hline \hline
\end{tabular}

Table 3. Hermetry form $H_{16}$ stands for the Higgs field(s), which is the only Hermetry form that does not contain subspaces $\mathrm{S}^{2}$ or $\mathrm{I}^{2}$, emphasizing the special role of this field permeating all physical space.

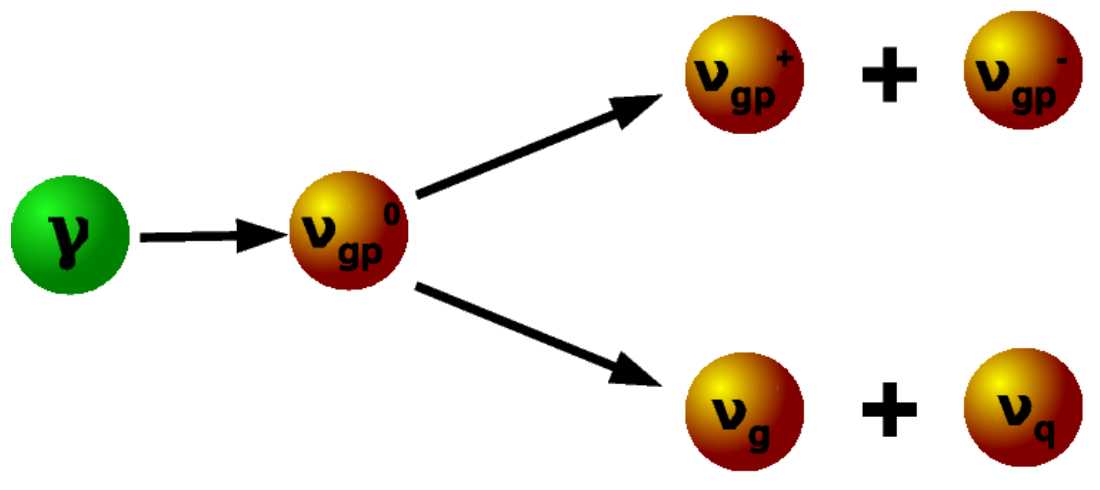

Figure 3. Hermetry form $H_{9}$ stands for the neutral gravitophoton, produced by photon conversion, which can decay via two different channels, depending on experimental conditions. The first one, upper branch, seems to take place in the generation of the axial acceleration field, the EHT experiment, see Sec. V. The second branch is assumed to occur in the gravitomagnetic experiments by Tajmar et al. and Graham et al. 
comparing Tables 1 and 2 actually are the neutral leptons $e^{0}, \mu^{0}, \tau^{0}$. At present, the mechanism of neutral lepton production is not known, but, as is directly obvious from Table 1 , the photon is the only particle that can be converted into a gravitophoton $v_{g p}^{0}$, which is the interaction boson for neutral leptons. It is therefore assumed that in the initial phase of the Universe, when huge numbers of photons were present, these particles possibly could have formed. Since they are not subject to electromagnetic interaction they might have life-times long enough to be stable and thus take part in galaxy formation. Furthermore, the experiments by Tajmar et al. can be explained assuming the production of gravitophotons by condensed matter phenomena, see Sec. IV] while accelerators are producing individual particles. However, the production of neutral leptons does not seem to occur in gravitomagnetic experiments, but instead it is assumed that NOM bosons occur in the form of gravitophotons and quintessence particles. In this regard, experiments by Tajmar et al. represent a completely novel class of phenomena involving non-ordinary matter.

In order to construct the physically meaningful set of metric sub-tensors that is, Hermetry ${ }^{d}$ forms, it is postulated that coordinates of internal spaces $\mathrm{S}^{2}$ (organization coordinates) or $\mathrm{I}^{2}$ (information coordinates) must be present in any metric sub-tensor to generate a Hermetry form.

Only metric tensors representing Hermetry forms are of physical relevance, and it is clear from their construction principle that all these tensors, derived from this underlying poly-metric tensor, are different. Consequently, their respective Gaussian curvatures, $K_{\ell}$, where $\ell$ denotes the index of the corresponding Hermetry form, must also be different. This is straightforward to observe, since Gaussian curvature is only a function of the first fundamental form (metric tensor components) as well as their first and second derivatives, but does not depend on the second fundamental form. Therefore, each Hermetry form $H_{\ell}$ determines its proper Gaussian curvature $K_{\ell}$, and thus curves space according to its own specific metric. Following the rule of $G R$ that interprets spacetime curvature as gravitational interaction, the appropriate Hermetry forms are thus interpreted as physical interactions, as shown in Tables 1 and 2 . each producing their independent spacetime curvature.

Having established the qualitative physical relationship between Hermetry forms and spacetime curvature, all physical interactions are connected to spacetime curvature, similar to $G R$, and in this sense physics has been geometrized.

\section{E. Symmetry Breaking Revisited}

In order to find the force that might be used as the propellantless propulsion principle, the nature of the novel physical interaction for the generation of gravitomagnetic fields needs to be determined. It is believed that the well known effect of spontaneous symmetry breaking is responsible, requiring, however, a completely different approach.

A superconductor or a ferromagnet are models for spontaneous symmetry breaking, that is the potential energy of the electron gas (superconductor) or the spin system (ferromagnet) changes abruptly at a critical temperature $T_{C}$, and the associated potential $V(\phi)$ is changing its shape from the left to the right curve as depicted in Fig. 4. As a result, a novel physical phenomenon occurs on the macroscopic scale.

For the subsequent discussion, the salient general characteristic features of symmetry breaking are listed below:

(i) Order parameter Spontaneous symmetry breaking is controlled by an order parameter, very often temperature. At a certain critical temperature, a completely novel and unexpected behavior of the physical system appears.

(ii) New Particles Spontaneous symmetry breaking is associated with the formation of a new type of particles that in, general, are strongly correlated. For instance, in superconductivity, Cooper pairs (two electron interaction, boson like) are formed.

(iii) Novel Physical Phenomenon At the macroscopic scale a novel, completely different physical behavior occurs. In superconductivity, electric resistivity becomes effectively zero at $T_{C}$.

When symmetry breaking occurs, e.g. by reducing the temperature of the system, the potential curve changes shape, moving from the left to the right picture in Fig. 4. The left shape is symmetric with respect to its single minimum (at $\phi=0$ ). For $m^{2}<0$, the minima correspond to $\phi= \pm v$. If spontaneous symmetry breaking sets in, the field settles at one of these minima for the self-interaction has lowered the energy of the ground state (or vacuum state). Thus the new shape (right picture) no longer reflects this symmetry, since the potential was shifted along the abscissa by $\pm v$. In other words, the symmetry has been broken spontaneously, i.e., by the physical system itself through its self-interaction potential triggered by the critical temperature. The original vacuum state is no longer the correct vacuum state, and the system has to assume a new vacuum state at $\phi_{0}= \pm v$ that is of lower energy. There is, however, an alternative to this mechanism. Instead, the system decides to regain its symmetry and particles of imaginary mass

\footnotetext{
${ }^{\mathrm{d}}$ Hermetry stands for the physical meaning of geometry, combined from hermeneutics and geometry
} 
are generated. It is postulated that this is the mechanism of generating imaginary particles of real charge whose subsequent conversion into gravitophotons is deemed to be responsible for the generation of the large observed gravitomagnetic fields.

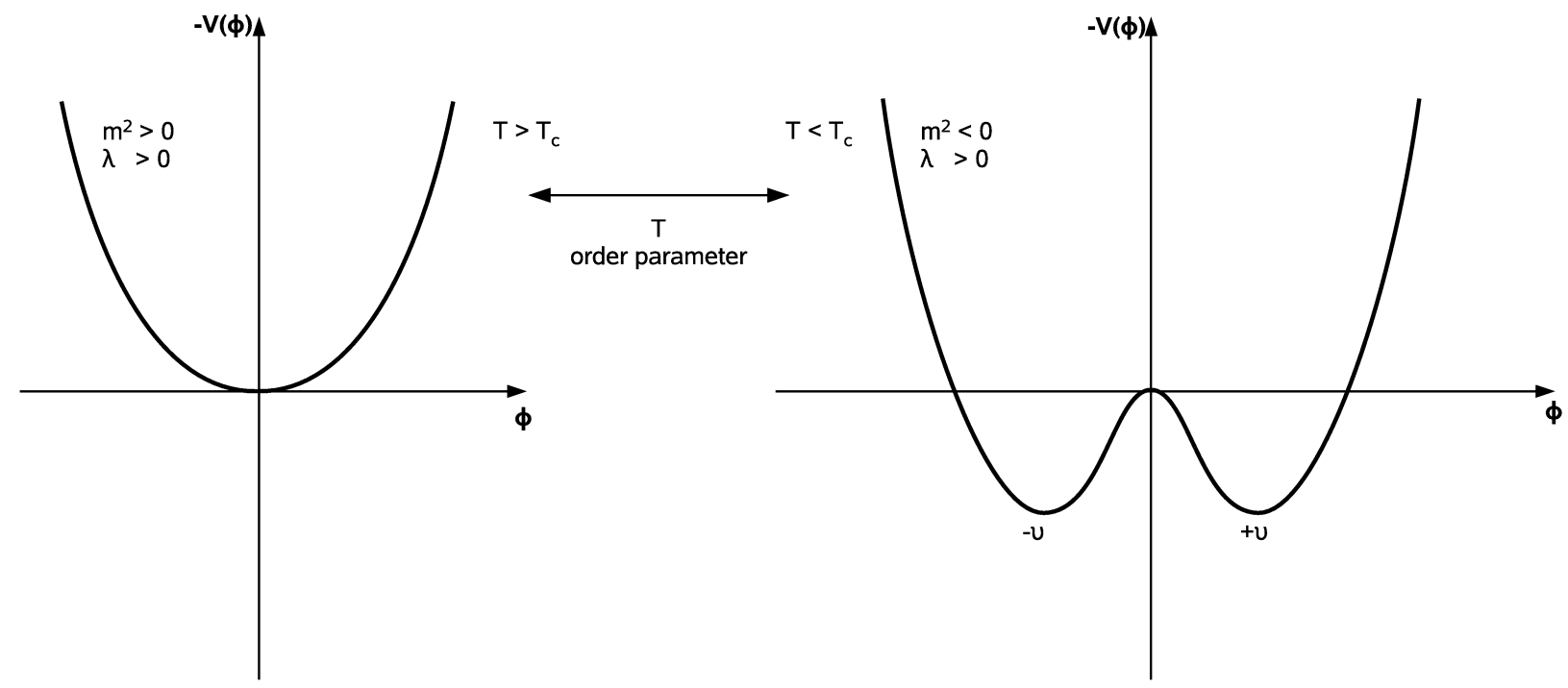

Figure 4. Symmetry Breaking Mechanism. The shape of the left potential function (depicted is $-V$ on the ordinate, $m^{2}$ and $\lambda$ have the same sign) displays a unique ground-state (or vacuum state), which is defined as the state that possesses the lowest energy. This potential belongs to a particle of mass $\boldsymbol{m}$ that is described by a Klein-Gordon field. At a critical temperature, $T_{C}$, whose magnitude depends on the physical system (e.g., superconductivity, superfluidity, ferromagnetism, condensed matter phenomena etc. ), so called spontaneous symmetry breaking sets in.

\section{F. Gravitomagnetism: Slight Symmetry Breaking and Particles of Imaginary Mass}

It is surmised that spontaneous symmetry breaking is instrumental for the laboratory generated large gravitomagnetic fields, since the magnitude of these fields suggests a coherent macroscopic phenomenon similar to superconductivity or ferromagnetism.

The canonical symmetry breaking mechanism does not seem to be applicable, since eventually a gravitational field is produced from a small rotating mass. Therefore, a different process than spontaneous symmetry breaking should take place, termed gravitomagnetic symmetry breaking (GSB), since it needs to account for the mechanism of first creating charged particles of imaginary mass from which the final gravitational field has to be produced. To

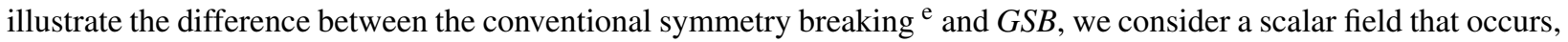
for instance, in ferromagnetism. It describes the potential energy of the spin system formed by the individual atoms. Replacing classical coordinates $q_{i}$ by scalar fields $\phi$ gives the general formulation of the Lagrangian

$$
\mathscr{L}=\partial_{\mu} \phi \partial^{\mu} \phi-V(\phi) \text { with } V(\phi)=-\frac{1}{2} m^{2} \phi^{2}-\frac{\lambda}{4 !} \phi^{4} .
$$

${ }^{\mathrm{T}}$ The first term of the Lagrangian describes kinetic energy, the second term the potential energy (harmonic oscillator) $\mathrm{g}$ and the third term stands for the self-interaction energy that physical systems exhibit in case of spontaneous symmetry breaking, i.e. the sign of $\lambda$ changes from positive to neagtive, see Fig 4 . In the case of ferromagnetism, the field variable $\phi$ is replaced by magnetization $\mathbf{M}$. Here, the coefficient of $M^{4}$ in potential $V(\phi)$ changes sign from minus to

${ }^{\mathrm{e}}$ For an excellent detailed introduction to this highly important phenomenon see Kaku, ${ }^{19}$ and also Schmüser, ${ }^{20}$ Zee, ${ }^{21}$ or Lahiri ${ }^{22}$ as well as the article by $\mathrm{Scheck}^{23}$.

${ }^{\mathrm{f}}$ There are no third order terms $\phi^{3}$, since $V(\phi)$ is invariant under the transformation $\phi \rightarrow-\phi$.

${ }^{\mathrm{g}}$ The so called Higgs field, represented by the symmetric Lagrangian as given above, would satisfy the Klein-Gordon equation, $\left(\square+m^{2}\right) \phi(x)=$ 0 , which describes a scalar particle of mass $m$, i.e., a particle with one Lorentz invariant, namely its four momentum $p^{\mu} p_{\mu}=m c^{2}$. For a particle with charge $q$, function $\phi(x)=\frac{1}{\sqrt{2}}\left(\phi_{1}(x)+i \phi_{2}(x)\right)$ has to be chosen complex. In the ground state $\left|\phi_{0}\right|$ the associated Noether current is given by $j^{\mu}=-2 q^{2}\left|\phi_{0}\right|^{2} A^{\mu}$. In case the electromagnetic interaction is associated with the symmetry breaking, the vector potential, $A^{\mu}$, hitherto represented by the massless photon, $\gamma$, must have acquired the real mass $M=\sqrt{2} q\left|\phi_{0}\right|^{20}$. Thus, spontaneous symmetry breaking has the capability to provide real physical mass to a former massless particle. 
plus at $T=T_{C}$, the Curie temperature, and thus the potential changes its shape, namely from the left to the right curve in Fig 4 Hence, $V(\phi)$ assumes a new minimum (negative) at $M_{0}$ (or $\phi=\phi_{0}$ ). The field is interacting with itself, which means for the superconducting state that the wave functions of the individual Cooper pairs are strongly correlated, forming a single macroscopic wave with $\psi_{C P}^{2}=n$ where index $C P$ denotes Cooper pairs and $n$ is their spatial density. In a ferromagnet there exists an interaction among the magnetic moments of the electrons of the $3 \mathrm{~d}$-orbits that are not closed.

For the explanation of spontaneous symmetry breaking according to quantum field theory (QFT) it is argued that the physical system is moving away from its original vacuum state (left curve in Fig. 4 and moves down the potential curve (right curve of Fig. (4) to the bottom of one of the wells to achieving a lower energy state. Consequently, the conventional interpretation of spontaneous symmetry breaking starts a Taylor expansion about the new minimum located at $\phi_{0}= \pm \sqrt{-6 m^{2} / \lambda}$, see also ${ }^{19}$. This means that a new field $\tilde{\phi}:=\phi-\phi_{0}$ is defined that now assumes its minimum at $\tilde{\phi}=0$. Now the potential expressed in terms of $\tilde{\phi}$ has the form

$$
V(\tilde{\phi})=-\frac{1}{2} m^{2} \tilde{\phi}^{2}+\frac{1}{6} \lambda \phi_{0} \tilde{\phi}^{3}+\frac{\lambda}{4 !} \tilde{\phi}^{4}
$$

which means that the origin of the coordinate system was shifted to the new vacuum and therefore the potential is no longer symmetric under the transformation $\tilde{\phi} \rightarrow-\tilde{\phi}$. Thus, the symmetry was spontaneously broken. In addition, as is mentioned in the footnote below, a novel particle of real mass mass has been created. So far the conventional interpretation of the role of spontaneous symmetry breaking.

Next, we present the spontaneous symmetry breaking mechanism, as suggested by $E H T$, considered responsible for the generation of the $\mathbf{B}_{g p}$ field. In order to explain the existence of these strong gravitomagnetic fields, the above mechanism does not work since electromagnetism is not the only interaction involved. Eventually, a gravitomagnetic field is measured. If we now suppose that in the Lagrangian, Eq. $3, \mathrm{~m}^{2}$ were negative, the new minimum of potential $V$ is given by $\phi_{0}= \pm \sqrt{-6 m^{2} / \lambda}$ and the expectation values of the vacuum state is not zero, but is given by

$$
\langle 0|\phi| 0\rangle=\phi_{0} .
$$

In the literature this potential is sometimes called the tachyon potential. If $m^{2}$ is negative, then $m$ is imaginary. The conventional interpretation is that the theory predicts particles of imaginary mass, moving at superluminal speed, called tachyons. Tachyons, however, have never been observed despite substantial experimental efforts. Quantum mechanically, one reinterprets the theory to mean that one has simply expanded around the wrong vacuum and the symmetry appears to be broken.

The salient characteristic features of GSB (Gravitomagnetic Symmetry Breaking) are stated below. Its application to the gravitational experiments (Sec. III) is detailed in Sec. IV] In order to explain GSB the two novel physical concepts derived from the Hermetry picture are utilized, namely the existence of three gravitational forces (Fig 2 ) and the existence of non-ordinary matter (Table $2 \mathrm{~h}^{\mathrm{h}}$

(i) GSB Order parameter $G S B$ symmetry breaking is controlled by temperature. At a certain critical temperature, denoted $T_{B}$, the cryogenic material exhibits a totally unexpected behavior such that extreme $\mathbf{B}_{g p}$ fields are produced. For instance, in the experiments at ARC a value of $T_{B}=15.9 \mathrm{~K}$ was found for the cryogenic rotating $\mathrm{Nb}$ ring.

(ii) GSB New Particles GSB symmetry breaking is associated with the formation of virtual electrons, $e_{I}$ and protons, $p_{I}$ of imaginary mass (in the form of imaginary quarks), generated by the GSB mechanism. These particles belong to NOM, see Table 2 , and do not exist in the current framework of ordinary matter given in Table 1

(iii) GSB Novel Physical Phenomenon On the macroscopic scale, the novel physical behavior is manifesting itself in the form of extreme $\mathbf{B}_{g p}$ fields.

\section{G. Energy and Momentum Conservation Revisited}

Relativistic space flight is not an option, since any space vehicle attaining velocities close to the speed of light can only achieve this at the expense of an infinite amount of energy, if the theory of special relativity (SR) was governing all physical laws. i

\footnotetext{
${ }^{\mathrm{h}}$ The following explanations are merely attempting to proposing the physical mechanism for the generation of the measured gravitomagnetic field. The mathematical analysis of this mechanism will have to demonstrate its viability.

${ }^{\mathrm{i}} \mathrm{SR}$ is experimentally very well established and thus is a validated physical theory. However, this does not exclude the existence of additional physical interactions as suggested by the gravitomagnetic experiments. Second, the range of applicability of SR is Minkowski space $M^{4}$, i.e., there
} 
Even if nonrelativistic flight of a spacecraft of mass $10^{5} \mathrm{~kg}$ at a speed of $10^{4} \mathrm{~km} / \mathrm{s}$ is considered, the kinetic energy to be provided is $5 \times 10^{18} \mathrm{~J}$. A $100 \mathrm{MW}$ nuclear reactor, providing the power for this spacecraft, would need $5 \times 10^{10}$ $\mathrm{s}$ or about 1,500 years to produce this amount of energy. This scenario is somewhat unrealistic, since the mass of the reactor was not accounted for. Clearly, physical laws governing this type of space propulsion are not suitable for real space travel.

Therefore, as an alternative means to space propulsion, already in 1960 Corliss $^{2}$ investigated the use of natural force fields, focusing on propulsion systems that do not rely on the expulsion of mass and energy. In other words, Corliss already discussed a propellantless propulsion mechanism. However, his discussion was restricted to conventional gravitational, magnetic (e.g., using the Earth magnetic field for propulsion), and electric (nothing has been observed) fields in space. He also discussed the modification of gravity, but Tajmar et al. ${ }^{24}$ have already shown that even if modified gravitational laws existed, their usage for space propulsion is negligible. The conclusion reached by Corliss was that nothing has been uncovered to allow any action-at a-distance force field for space propulsion in interplanetary or interstellar space.

Concerning the production mechanism of gravitophotons, as long as $T<T_{C}$, gravitophotons will be produced by the mechanism described in Sec. IV] and a strong $\mathbf{B}_{g}$ field is observed. As discussed in Sec. III C, there is evidence from ARC experiments that the temporal variation of this field leads to a gravity-like (acceleration) field and thus to a novel force. The important fact is that in the generation of this force through the decay of the gravitophoton both particles from $O M$ (graviton positive energy density) and NOM (quintessence particle, negative energy density). The total energy needed in generation these two particles is therefore zero. Gravitons interact with the space vehicle and cause its acceleration while the momentum of the quintessence particle is not felt by the space vehicle but by the surrounding spacetime.

\section{Experiments for the Laboratory Generated of Gravitational Fields}

In this section, we are dealing with gravitational fields that are gravitomagnetic (1/s) or gravity-like (acceleration, $\mathrm{m}^{2} / \mathrm{s}$ ) fields, generated in the laboratory by a cryogenic rotating mass below a certain critical temperature. In comparison to $G R$, these gravitomagnetic fields are about 18 orders of magnitude larger than those predicted by the frame dragging effect of $G R$. Therefore, if current experiments are confirmed, the physics of these fields must be outside $G R$. The physical mechanism underlying this effect, according to $E H T$, will be discussed further in Sec. IV] It was, however, shown in ${ }^{9}$ that, if experimental measurements are correct, none of the currently known four physical interactions would be sufficient to account for these novel physical phenomena.

\section{A. Present Experimental Basis for Gravitational Fields}

Recently Tajmar et al. carried out a comprehensive series of different gravitomagnetic experiments ${ }^{33}$ termed A, B, and C. The difference between the three experimental setups is as follows.

Setup A In setup A the sensor vacuum chamber is directly above the cryogenic spinning ring. There is a very thin sheet of insulating material, MLI between the vacuum chamber and the sample holder containing the rotating ring. However, there is no MLI between the upper surface of the ring and the sensor vacuum chamber.

Setup B In setup B the vacuum sensor chamber is inserted in a stainless steel container, and thus the gyroscope could be shielded to some extent from the gravitomagnetic field of the rotating ring.

Setup C In experiment C, the stainless steel shielding of Setup B is replaced by an MLI made container of about 1 cm thickness.

Canterbury Experiment In the experiments by Graham et al. a large laser ring gyro is used, and the resulting gravitomagnetic field is measured outside the vacuum sensor chamber. However, the sensitivity of this laser gyro was two orders of magnitude lower compared to the gyro used in Setup B and C.

is no acceleration of the space vehicle. This does not necessarily exclude the existence of other physical spaces, which a space vehicle might be able to enter and leave under certain physical conditions. Third, spacetime is discrete (quantized version of spacetime), and as such cannot be Lorentz invariant.

${ }^{\mathrm{j}}$ Multi Layer Insulation, responsible for the gold foil color in spacecraft insulation, is used for thermal insulation in vacuum by minimizing radiation losses. A single layer has a thickness of approximately $10^{-5} \mathrm{~m}$. It is made from amber colored Kapton (plastic), having on one side a silvery aluminum coating for high thermal reflection in the infrared. Other material combinations like Mylar (plastic) and silver are also used. 
Gravity-Probe B Gravity-like fields might have been observed also in the NASA-Stanford Gravity Probe B Experiment and were already discussed in ${ }^{9}$. According to EHT, part of the observed gyro anomaly should be due to the existence of gravity-like fields, and should not be completely explainable by electrostatic forces (patch effect).

\section{B. Gravitomagnetic Field Experiments at Austrian Research Centers}

The experimental setups $\mathrm{A}, \mathrm{B}$, and $\mathrm{C}$ have resulted in highly varying gravitomagnetic field strengths as can be seen from Tables 4, 5. There are several points to be noted.

In Setup A, the evaporating helium escapes through the duct on the upper left hand side and gaseous helium, produced by evaporation of the liquid helium from the cyrostat, is streaming along the walls of the stainless steel vacuum sensor chamber housing the gyros. The shaft, see middle axis, is a massive stainless steel rod of $20 \mathrm{~mm}$ radius or, as used in the most recent experiments, ${ }^{33}$ was replaced by a tubular structure, in order to strengthen the gyro support. There is an MLI layer, (thin Al foils), half the thickness of the one employed in Setup C, insulating the sample holder from the vacuum sensor chamber. There is no MLI material in the gap directly above the ring and the sensor chamber. The measurements from this Setup are shown in Table 4 . The gyros are housed in a $2 \mathrm{~mm}$ strong stainless steel casing. The gyro support is an Al plate of thickness 2-3 mm.

In Setup B the sensor chamber is embedded in a steel vessel so that ring and chamber are further separated by a 5 $\mathrm{mm}$ distance. Thus the contact of the evaporating helium with the sensor chamber is reduced. However, some liquid helium is vented through openings (not visible in the left figure) in the flanges connecting the cryostat and insulation vessel, in order to prevent ice formation along the sensor chamber walls. The velocity of this helium gas should be small, since it passed through the stack of cooling metal sheets. The major part of evaporating helium leaves the cryostat through the helium venting tube on the upper left. Here, a single, but substantially more sensitive gyro is used. This gyro has a larger footprint than the ones in Setup A.

In Setup $C$ the evaporating helium stream does not stream along the sides of the vacuum sensor chamber. Compared to experiment B, the gyro chamber is thermally insulated from the helium by an additional layer of MLI. The shaft, see middle axis, is a tubular structure. Gaseous helium, produced by evaporation of the liquid helium from the cyrostat, is now streaming along the sides of the inset cryostat, made from MLI, which replaces the insulating stainless steel chamber of setup B. The measurements from these two variants are shown in Table 4 The gyro support is an $\mathrm{Al}$ plate of thickness 2-3 mm. The liquid helium in the cryostat does not take part in the rotation, since the filling level in general remains below the sample holder, except for the results shown in the first yellow row of Table 5 .

Comparing the gyro results as shown in Table 4 , it is obvious that there are substantial differences in the clockwise gyro signals, and it also might seem that there are inconsistencies in the measured counter-clockwise signals. However, as shown in the rightmost column, the ratio of the CW/CCW signals, within their specified measured uncertainties, always assumes one of the integer values $1,-1,5$, and -5 as predicted in. At present, it cannot be predicted under which experimental conditions a specific ratio is seen. These values should occur according to EHT, and are derived considering the partial terms of the corresponding Hermetry forms. The last row (green) shows the results by Graham et al. ${ }^{32}$. Results of Graham were obtained by utilizing the high precision ring laser gyro UG2, operated by the Canterbury Ring Laser Group that has dimensions $21.0 \mathrm{~m} \times 39.9 \mathrm{~m}$. In this experiment, due to its size, the gyro is operated outside the cryostat, and therefore any influence of evaporating helium on gyro vibrations can be excluded. The change in sign (compared to results of Tajmar et al.) of the $\mathbf{B}_{g}$ field can directly be explained from the fact that the ring laser gyro saw the downward component of the dipole gravitomagnetic field component, since the measurement location was outside the disk.

In setups B and C the angular frequency was limited to $\omega=100 \mathrm{rad} / \mathrm{s}$ instead of $\omega=420 \mathrm{rad} / \mathrm{s}$ in setup A (see Table 47. Comparing the results of setups B and C, it is obvious that there is substantial difference in the magnitude of the gyro signals. Furthermore, if the $5 \sigma$ rule is employed that, in order to have a valid gyro signal, the measured signal magnitude must be at least five larger than the standard deviation, only the measured results of the first red row would qualify as a valid signal. In addition, even the $\mathrm{CCW}$ value in this row might have to be considered a null signal. From ${ }^{33}$ all uncertainties are restricted to one digit accuracy, no information is available on the second digit. Applying the same rule to the yellow measurements, all measured results should be disqualified, except perhaps the first value in the first row. However, if the uncertainty were about $10 \%$ larger than specified, this result also would have to be considered a null result. Since the uncertainties in setup $\mathrm{C}$ are already one order of magnitude less than the ones in setup B, it is not clear how accurate they were determined. In other words, it could be that all results in setup $B$ and $C$ need to be discarded. There seems to be substantial uncertainty to interpret the signals of setup $\mathrm{C}$ in such a way that only the rotating liquid helium is giving a signal, while the gravitomagnetic signals of the $\mathrm{Al}-\mathrm{Al}$ and $\mathrm{Nb}-\mathrm{Al}$ are counted as null. If the casing of setup $\mathrm{C}$ works similar to a gravitomagnetic cage, all signals are being drastically 


\begin{tabular}{|c|c|c|c|c|c|}
\hline Material & $\begin{array}{c}\text { Sample } \\
\text { Holder }\end{array}$ & Geometry & $C W$ & $C C W$ & $C W / C C W$ \\
\hline $\mathrm{Nb}$ & $\mathrm{Al}$ & ring & $3.2 \pm 0.5$ & $-0.4 \pm 0.3$ & -5 \\
\hline YBCO & $\mathrm{Al}$ & ring & $5.3 \pm 0.2$ & $-1.2 \pm 0.1$ & -5 \\
\hline $\mathrm{Al}$ & $\mathrm{Al}$ & ring & $3.8 \pm 0.2$ & $-0.7 \pm 0.3$ & -5 \\
\hline $\mathrm{Nb}$ & $\mathrm{Al}$ & ring & $5.7 \pm 0.4$ & $4.8 \pm 0.5$ & 1 \\
\hline YBCO & $\mathrm{Al}$ & ring & $3.1 \pm 0.4$ & $0.3 \pm 0.4$ & 5 \\
\hline Teflon & Teflon & ring & $3.1 \pm 0.4$ & $-0.5 \pm 0.5$ & -5 \\
\hline- & - & no sample & $-0.1 \pm 0.2$ & $-0.3 \pm 0.1$ & - \\
\hline $\mathrm{Pb}$ & $\mathrm{Pb}$ & disc & $-5.3 \pm 8.5$ & $37.7 \pm 13.2$ & -5 \\
\hline
\end{tabular}

Table 4. The three (yellow) rows depict the measured values for Setup A, compiled from ${ }^{30}$.The following four (red) rows show the gravitomagnetic field measurements (shown is the so called coupling factor $\times 10^{8}$ at temperatures $T=4-6 \mathbf{K}$, that is defined as gyro-signal per angular frequency $\left(\mathrm{rad} \times \mathrm{s}^{-1} / \omega\right)$ for Setup $\mathbf{A}$ as reported in Tajmar et al. ${ }^{33}$. The last row, green, shows the measurements obtained from Graham et al. where a disk was used and measurements were done outside the cryostat employing a large ring laser. Note that these measurements do not satisfy the $5 \sigma$ rule. Measured magnitudes of the $\mathbf{B}_{g}$ depend on the direction of rotation. The last column shows the theoretically calculated ratio.

reduced. However, as shown again in the rightmost column, the ratio of the CW/CCW signals, within their specified measured uncertainties, seems to assume one of the integer values $1,-1,5$, and -5 as already mentioned in Table 4 .

Assessment of Gravitomagnetic Field Experiments:

- The measured CCW signals for Setup A in Table 4 reveal a change in sign.

- The noise levels in Setup A are in the range of the largest signal measured in Setup C.

- If the $5 \sigma$ rule is strictly applied, the results in Table 5 would have to be discarded and the same holds for several measurements of Table 4

- On the other hand, the gyros report sign changes in the signals, i.e. they actually seem to see a signal, which is no random.

\section{Gravity-Like Field Experiments at Austrian Research Centers}

In these experiments a gravitomagnetic force is produced by a ring, disk or sphere that needs to be accelerated or decelerated in order to obtain a gravity-like field in the rotational plane directed in the circumferential direction. The gravity-like field acts against the original mechanical acceleration or deceleration field. This experiment, if confirmed, would have seen a novel physical force. Application to a novel technology, however, is not at all straightforward. First, the process of acceleration or deceleration can only be sustained for a very limited period of time. Any acceleration eventually must be followed by a deceleration, so that over a large enough averaging period in time the gravity-like field is zero. Second, the field is in the circumferential direction, making it difficult to use it as propulsion source.

\section{Physical Mechanism of Gravitational Experiments}

As was shown $\mathrm{in}^{9,12}$ the neutral gravitophoton that causes the gravitomagnetic force can decay into a graviton and quintessence particle, as is assumed to be the case in the ARC and Graham experiments as well as for GP-B. There should, however, exist a second decay avenue, namely into a positive (repulsive) gravitophoton and negative (attractive) gravitophoton, which should take place in the proposed experiment for the axial acceleration field (Fig. 


\begin{tabular}{|c|c|c|c|c|c|}
\hline \multicolumn{6}{|c|}{ Gravitomagnetic Measurements for Setup B and C by Tajmar et al. } \\
\hline Material & $\begin{array}{c}\text { Sample } \\
\text { Holder }\end{array}$ & Geometry & $C W$ & $C C W$ & $C W / C C W$ \\
\hline St.-Steel & St.-Steel & ring & $3.4 \pm 0.5$ & $-4.7 \pm 0.9$ & -1 \\
\hline $\mathrm{Al}$ & $\mathrm{Al}$ & ring & $2.1 \pm 0.8$ & $-2.2 \pm 0.5$ & -1 \\
\hline $\mathrm{Al}$ & - & disc & $0.3 \pm 0.1$ & $-0.3 \pm 0.1$ & -1 \\
\hline $\mathrm{Al}$ & $\mathrm{Al}$ & He cup fins & $0.17 \pm 0.03$ & $0.01 \pm 0.09$ & -1 \\
\hline $\mathrm{Al}$ & $\mathrm{Al}$ & ring & $-0.03 \pm 0.03$ & $-0.04 \pm 0.04$ & 1 \\
\hline $\mathrm{Nb}$ & $\mathrm{Al}$ & ring & $-0.12 \pm 0.05$ & $-0.12 \pm 0.08$ & -1 \\
\hline $\begin{array}{l}\text { YBCO } L_{2} N_{2} \\
T=77-90 \mathrm{~K}\end{array}$ & $\mathrm{Al}$ & ring & $0.0 \pm 0.01$ & $0.01 \pm 0.01$ & - \\
\hline
\end{tabular}

Table 5. The three (red) rows show the coupling factor $\times 10^{8}$ at temperatures $T=4-6 \mathrm{~K}$ for Setup $\mathbf{B}$ and $\mathrm{C}$ as reported in Tajmar et al. ${ }^{33}$. The following three (yellow) rows depict the measured values for Setup $\mathrm{C}$, compiled from ${ }^{30}$. The green row shows measured results at the temperature of liquid nitrogen. Since the effect is associated with a phase transition at low cryogenic temperatures a zero gravitomagnetic field is to be expected.

6. In the experiment by Tajmar et al. the gravity-like field $\mathrm{d}^{\mathrm{k}}$ is in the circumferential direction and needs a time varying neutral gravitophoton field, see ${ }^{9}$. For the axial field generation, the time varying differential operator is replaced by a spatially varying operator, which should lead to a completely different nature of the gravity-like force that is much more amenable to space propulsion purposes, since it should be directed along the axis of rotation.

In both cases the energy extracted from the vacuum is zero, since graviton and quintessence particles have positive and negative energy densities, respectively. If only the energy of the gravitons were measured, it should seem that energy conservation is violated. However, this would be a clear sign that the energy budget is not complete, because the negative energy density of the quintessence particle was not accounted for. In the axial field experiment, the total energy taken from the vacuum is also 0 . The two gravitophoton fields have opposite energy densities and add up to zero energy density. The important role of the vacuum field is (spacetime) that it allows this type of pair creation. It seems to act as catalyst for such a reaction.

First, the list of particles is given that might be involved or being created during the physical processes that are taking place in the generation of gravitomagnetic or gravity-like fields.

(i) $e$ the electron is assumed to carry a negative charge,

(ii) $p=u u d$ proton from crystal lattice comprising three quarks ( $\mathrm{u}$ has charge $+2 / 3 e$ and d quark $-1 / 3 e$ ),

(iii) $v_{g}$ graviton,

(iv) $e_{I}$ imaginary electron, same charge as the electron,

(v) $q_{I}$ three imaginary quarks generated within protons of the crystal lattice at cryogenic temperature together $e_{I}$ satisfying charge conservation,

(vi) $v_{q}$ quintessence particle,

(vii) $e_{I}^{B}:=e_{I}+e_{I}$ imaginary electron-boson pair from two electron interaction, which is similar to $e^{B}:=e+e$ electronboson pair. According to experiment, the gravitomagnetic field was already measured at temperatures around $25 \mathrm{~K}$, somewhat above the critical temperature of $\mathrm{Nb}(9.2 \mathrm{~K})$, and hence $e^{B}$ pairs might not be needed,

(viii) $v_{g p}^{0} \rightarrow v_{g}+v_{q}$, neutral gravitophoton generated in the gravitomagnetic experiments by Tajmar et al.,

\footnotetext{
${ }^{\mathrm{k}}$ The experiments by Tajmar et al. and Graham et al. are also discussed by Hathaway, Chap. 5 in $^{3}$. Data from the two experiments show similarities, in particular, a signal strength dependence on the direction of rotation, though measurement techniques were completely different. One of the conclusions is that further independent experiments need to be performed.
} 
(ix) $v_{g p}^{0} \rightarrow v_{g p}^{+}+v_{g p}^{-}$, neutral gravitophoton generated in vertical gravity-like field as proposed from $E H T$.

Second, in all experiments generating gravitomagnetic or gravitoelectric (acceleration) fields the production of imaginary matter is assumed, whose subsequent conversion into a gravitomagnetic or gravitoelectric field is deemed responsible for the enormous frame dragging like effect, which is about 18 orders of magnitude larger than that of GR and therefore outside GR. The production mechanism for imaginary matter (PIM) is supposed to proceed in the following stages:

1. PIM [ $q_{I}$ generation in $\mathrm{Nb}$ ring ] at a critical cryogenic temperature (material dependent), a phase transition occurs and imaginary quarks $q_{I}$ within the protons of the crystal lattice are produced. The three $q_{I}$ quarks are the source of an imaginary vector potential $\mathbf{A}_{I}$. Neutrons do not contribute to $e_{I}$ generation, since they do not carry charge,

2. PIM [ $e_{I}$ generation in $\mathrm{Nb}$ ring ] at the same time imaginary electrons are produced, according to the mechanism of symmetry breaking, described in Section. IIE, and are supposed to appear as free imaginary electron gas,

3. PIM [ $e_{I}^{B}$ generation in $\mathrm{Nb}$ ring ] imaginary electron-boson pairs may be formed as in conventional BCS theory, due to the exchange of lattice vibrations, also known of phonons. 1]

Third, three different types of gravitational experiments are discerned in the generation of the gravitomagnetic or gravitoelectric fields. The different options are listed below:

1. [Gravitational Experiment 1: Gravitomagnetic field] Rotating the cryogenic $\mathrm{Nb}$ ring at constant angular velocity $\omega_{N b}$ produces a gravitomagnetic field $\mathbf{B}_{g p}$ only. This field was measured by Tajmar et al. and Graham et al., causing a rotation (twisting) of spacetime, and also seems to (partially) cause a misalignment of the gyro axes in the GP-B experiment. No acceleration field is present.

2. [Gravitational Experiment 2: horizontal acceleration field ] Using a mechanical force to accelerate the cryogenic $\mathrm{Nb}$ ring that is, variation of $\omega_{N b}$, generates a tangential (or circumferential) gravity-like (acceleration) field $\mathbf{g}_{g p}$ located in the plane of the ring in circumferential direction, acting against its origin (i.e., the mechanical acceleration), following some kind of Lenz rule, as measured by Tajmar et al.

3. [Gravitational Experiment 3: vertical acceleration field] Employing a (special material) superconducting solenoid to induce a magnetic induction field in the cryogenic (superconducting) ring (or disk) should generate a vertical gravity-like acceleration field (GE 3, see Fig 6) acting along the axis of rotation of the $\mathrm{Nb}$ ring (or disk), predicted by EHT. In this experiment the ring (or disk) is rotating at constant speed, in contrast to GE 2 .

Fourth, there seem to exist two coupling mechanisms to generate gravity-like fields, termed fermion and boson coupling.

Fermion coupling: Imaginary charges are created by radiation coupling in form of virtual electron-positron pairs and their interaction with the complex Higgs field ${ }^{38}$. This type of coupling requires very strong magnetic induction fields, and thus is no longer pursued.

Boson coupling: Experiments of Tajmar et al..$^{29-31}$. Imaginary electron pairs (boson like) $e_{I}^{B}$ (Hermetry form $H_{11}\left(\mathrm{~T}^{1} \times \mathrm{S}^{2}\right)$ ), possibly created by some kind of two-electron interaction (similar to conventional BCS theory), are interacting with the imaginary vector potential $\mathbf{A}_{I}$ from the imaginary quarks $q_{I}$ in the lattice, resulting in real physical interaction. The technical requirements for achieving boson coupling are less demanding than for fermion coupling. For instance, the strength of magnetic induction field is substantially lower for boson coupling.

Fifth, the description of the physical processes that are taking place in the generation of gravitomagnetic and gravitoelectric fields are presented. In a step by step fashion, the sequence of physical phenomena as they occur in the various stages of the experiments are presented.

\footnotetext{
${ }^{1}$ It is however assumed that there is no interaction between charged real and imaginary particles that is, the generic many-particle Hamiltonian for a metal $H=H_{e}+H_{i}+H_{e i}$ is not modified by the presence of terms like $H_{e e_{I}}$ or $H_{i e_{I}}$, but a term $H_{e_{I} e_{I}}$ might be added.
} 
1. Cryogenic temperature Nb-ring: In all experiments, the rotating $\mathrm{Nb}$ ring is cooled down to cryogenic temperatures. Gravitomagnetic or gravity-like fields are observed only below a certain critical temperature, $T_{G}$, which is generally higher than the critical temperature, $T_{S}$, for superconductivity. Therefore, the mechanism that triggers the onset of gravitomagnetic fields, has to be associated with its proper phase transition where temperature is the order parameter.

2. Constant rotation of $\mathbf{N b}$ ring and $\mathrm{Al}$ sh: In Experiment 1, both the $\mathrm{Al}$ sample holder (sh) and its sample, namely the $\mathrm{Nb}$ ring that is mechanically fixed to the $\mathrm{Al} \mathrm{sh}$, are rotated at constant angular speed $\omega_{N b}$. It is known that, reaching a critical temperature, $T_{G}$, spontaneous symmetry breaking occurs, and the cryogenic material (i.e., $N b$ ring, Al sh, and also the liquid He itself) will be in the gravitomagnetic state as denoting this kind of phase transition. As it seems, the superconductive state is not necessary for the gravitomagnetic state, but perhaps it is a sufficient condition, at least for some materials.

3. Cryogenic temperature Al-sample holder: Superconductivity comprises two-electron interactions that is, producing electron-boson (Cooper) pairs, $e^{B}$, as described under 3. PIM. The gravitomagnetic state is similar, such that by employing 2. PIM, imaginary electron-boson pairs, $e_{I}^{B}$, are generated. In agreement with 1. PIM, imaginary quarks, $q_{I}$, are generated by the protons in the crystal lattice in the $\mathrm{Nb}$ ring that are fixed to their locations of origin. According to 2. PIM, imaginary electron pairs $e_{I}^{B}$ are also generated in the $\mathrm{Al}$ sample holder.

4. Conversion from electromagnetic to gravitomagnetic field : The imaginary electron pairs that are moving with the crystal lattices of the rotating $\mathrm{Nb}$ ring and $\mathrm{Al}$ sh, interact with the imaginary vector potential, $\mathbf{A}_{I}$, from the stationary imaginary quarks. The interaction of these virtual imaginary particles causes a real physical effect, namely, since no electromagnetic effect is observed, instead conversion into a gravitomagnetic field must have taken place by the production of neutral gravitophotons $v_{g p}^{0}$, which are considered to be the field quanta of the observed gravitomagnetic field $\mathbf{B}_{g p}$.

$$
\mathbf{B}_{g p}^{C W}=20 \pi \frac{1}{\sqrt{\alpha_{g p}}} \frac{G}{c^{2}} \frac{m_{e}}{m_{p}} \rho A \omega_{N b}
$$

where $\rho$ is the combined density of $\mathrm{Nb}$ ring $\left(8.57 \times 10^{3} \mathrm{~kg} / \mathrm{m}^{3}\right)$ and $\mathrm{Al}$ sample holder $\left(2.70 \times 10^{3} \mathrm{~kg} / \mathrm{m}^{3}\right)$ and $A=2 \pi \times 7.5 \times 10^{-2} \times 6 \times 10^{-3}=2.83 \times 10^{-3} \mathrm{~m}^{2}$ is the area of the ring, see Fig. 5. For the actual magnitude of the $\mathbf{B}_{g p}$ field the masses of the ring and sample holder (and thus the distribution of mass) is of importance as well as the material. The field scales linearly with the angular velocity $\omega_{N b}$ of the ring, at least over a certain range of $\omega_{N b}$.

\section{Gravitational Space Propulsion Device}

In GME 1 and 2 that are specifically designed for the generation of gravity-like fields, the gravitational force is acting in the plane of rotation in circumferential direction, opposing the original acceleration of the ring or disk, following some kind of Lenz rule. The same holds true for GME 3, GP-B, which was designed to measure the Lense-Thirring effect.

Therefore, $E H T$ was used to investigate whether a technically more convenient gravity-like field can be generated whose force component is along the axis of rotation, while the speed of rotation remains constant. The existence of such a field is based on the fact that spatial and temporal coordinates should be of the same physical quality.

Considering the experimental setup by Tajmar et al. that comprises an aluminum ( $\mathrm{Al}$ ) sample holder (the $\mathrm{Nb}$ ring) and cryogenic rotating $\mathrm{Nb}$ ring fixed to the sample holder, and thus rotating at the same angular velocity. Two acceleration components are generated: one in the radial $r$ direction of the disk, and the second one in the $z$ - direction. The first one, has to be absorbed by the structure of the configuration, thus creating a mechanical load. The component in the $\mathrm{z}$-direction, responsible for the acceleration field, is given by

$$
B_{g ; z}=k k_{N b} k_{A l} \frac{1}{\pi} \frac{m_{e}}{m_{p}} \omega_{I} ; a_{z} \hat{\mathbf{e}}_{\mathbf{z}}=\frac{v_{s h}^{2}}{c} B_{g ; z}\left(\hat{\mathbf{e}}_{\theta} \times \hat{\mathbf{e}}_{\mathbf{z}}\right) \times \hat{\mathbf{e}}_{\theta}
$$

and $\omega_{I}$ is the angular velocity of the imaginary electron pairs, $v_{s h}$ denotes the mechanical velocity of the rotating sample holder, and $B_{g ; z}$ is the component of the gravitophoton field $\mathbf{B}_{g}$ (dimension 1/s) in the $z$-direction. It should 


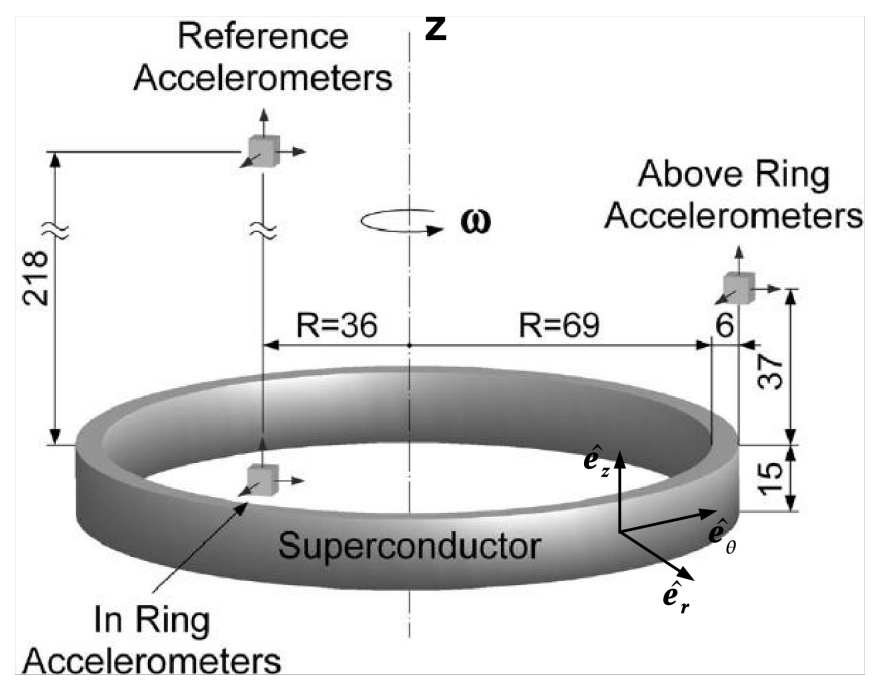

Figure 5. The picture shows the $\mathrm{Nb}$ ring as utilized in the experiments by Tajmar et al.

be noted that the magnitude of the gravitomagnetic field $\mathbf{B}_{g}$ does not depend on the mechanical angular velocity at which $\mathrm{Al}$ sample holder and $\mathrm{Nb}$ ring are moving. In contrast to Fermion coupling, ref., ${ }^{38}$ experimental requirements are substantially lower.

The following assumptions were made for the experiment producing the vertical gravity-like field : $N=10$, number of turns of the solenoid, current of about $13.6 A$ (needed to calculate $B_{z}$ ), diameter of solenoid $0.15 \mathrm{~m}$, and $v_{\theta}^{T}=50 \mathrm{~m} / \mathrm{s}$. The disk should be placed directly above the solenoid to produce a magnetic field in z-direction only. This experiment should give an acceleration field of about $\mathbf{g}_{g p}=6 \times 10^{-3}$ g $\hat{\mathbf{e}}_{z}$, which is an appreciable field acting directly above the rotating disk.

From these numbers it seems to be feasible that, if our theoretical predictions are correct the realization of a space propulsion device that can lift itself from the surface of the Earth is within current technological limits.

For a more realistic propulsion device in order to generate a force of $1.98 \times 10^{6} \mathrm{~N}$, a mass of $3.15 \times 10^{3} \mathrm{~kg}$ and a rotation speed of $200 \mathrm{~m} / \mathrm{s}$, a coil of $1 \mathrm{~m}$ diameter with 2,500 turns and a current of $13.6 \mathrm{~A}$ was calculated. The cross section area of the coil was determined to be about $2.5 \times 10^{-2} \mathrm{~m}^{2}$. These numbers will be recomputed in our forthcoming review article. All trip times given in $^{38}$ remain unchanged, but as can be seen from the specifications above, technical requirements were substantially reduced and should be feasible employing current technology. The reason for this change is boson instead of fermion (vacuum polarization) coupling.

\section{Conclusions and Future Activities}

Since 2002 ideas of a geometric approach for describing physical interactions, termed Extended Heim Theory $(E H T)$, were published. This approach predicts six fundamental physical interactions, three gravitational fields, electromagnetism as well as the weak and strong interactions ${ }^{9,13,14}$. In EHT gravitation can be both attractive and repulsive. EHT also predicts the existence of virtual particles of imaginary mass, responsible for the conversion of electromagnetic energy into gravitational energy. In addition to the existence of ordinary matter (fermions and bosons), non-ordinary matter in the form of above virtual particles of imaginary mass as well as stable neutral leptons should exist, which might be accountable for dark matter.

Numerous experiments by Tajmar et al. at ARC Seibersdorf carried out since 2003, and first published in 2006, report on the laboratory generation of gravitomagnetic as well as gravity-like fields. The gravitomagnetic effects measured were about 18 orders of magnitude larger than predicted by the so called Lense-Thirring effect of GR. In other words, the rotating niobium ring, having a mass of some 100 grams utilized by Tajmar, produces a frame dragging effect similar to the mass of a white dwarf ${ }^{9}$. These experiments were repeated by Graham et al. ${ }^{32}$ in 2007 , and more recently Tajmar et al. ${ }^{31}$ provided a comparison between the two experiments. If the experiments of Tajmar and Graham are correct, a similar effect should have been observed in the NASA-Stanford Gravity-Probe B experiment 


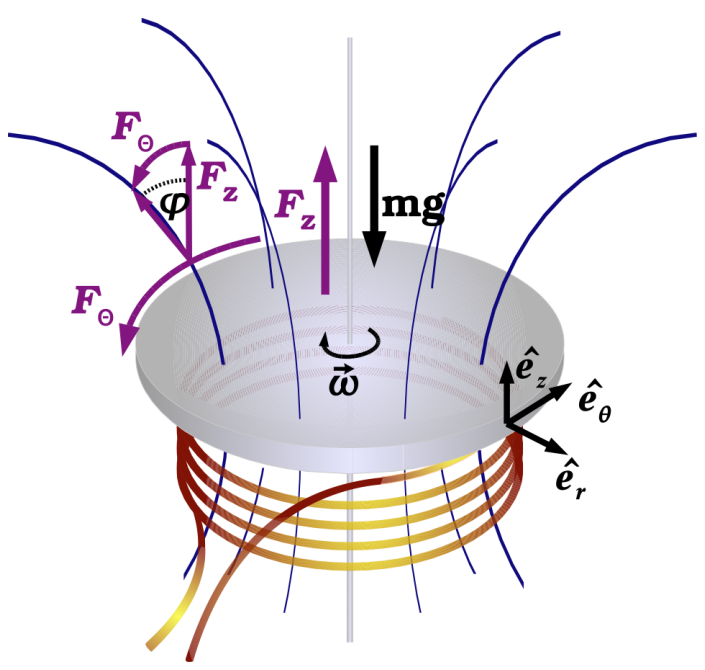

Figure 6. In this gravity-like field experiment the artificial gravitational field generated would be directed along the axis of rotation. The second component is in azimuthal direction and should accelerate the ring or disk. Therefore, energy needs not to be supplied to keep the angular velocity of the ring or disk constant. This experimental setup could serve as field propulsion device, if a non-divergence free field were generated (the physical nature of the gravity-like field is not known at present).

as was calculated in ${ }^{9}$. Indeed, a large gyro anomaly was observed in GP-B.

In all experiments a phase transition seems to have occurred at low temperatures (not necessarily at $T_{C}$, the critical temperature for superconducting, but possibly boson interaction took place (virtual imaginary particles). GR cannot be used to explain these phenomena, even if the full nonlinear Einstein field equations were used. The Lageos and GP-B experiments have clearly demonstrated that the inertial frame dragging effect, even from celestial bodies, is extremely small and within GR. These facts provide evidence for novel physics in the form of additional long range fundamental forces.

How to proceed? The experiments performed so far, if confirmed, will serve as demonstrators for the existence of a novel physical effect. However, in order to produce a propellantless space propulsion system, the experiment of Sec. II needs to be carried out. According to EHT, the effect should be large enough to be detectable with relatively simple measuring equipment, in contrast to the experiments performed so far, which need extremely sensitive equipment to measure a small effect, and thus are susceptible to background noise. Moreover, an axial field might directly lead to some kind of gravity control.

Furthermore, gravity-like fields most likely would lead to novel technologies in the general field of transportation, and thus should be of major interest to the public and, in particular, to industry. In addition, these fields might also be usable in energy generation leading to energy research that is highly relevant to the future.

\section{Acknowledgment}

The assistance by M.Sc. O. Rybatzki, Faculty Karl-Scharfenberg, Univ. of Applied Sciences, Salzgitter Campus in preparing the figures is gratefully acknowledged.

The authors are grateful to Dr. M. Tajmar, ARC Seibersdorf, Austria for providing measured data as well as for numerous comments regarding comparisons between EHT and gravitomagnetic experiments.

The authors are most grateful to Prof. P. Dr. Dr. A. Resch, director of the Institut für Grenzgebiete der Wissenschaft (IGW), Innsbruck, Austria for his support in writing this paper.

\section{References}

${ }^{1}$ Seifert, H. (ed.).: Space Technology, Wiley 1959.

${ }^{2}$ Corliss, R. C.: Propulsion Systems for Space Flight, McGraw-Hill 1960.

${ }^{3}$ Hathway, G.D.: Gravitational Experiments with Superconductors: History and Lessons, Chap. 5, in M.G. Millis, E.W. Davis (eds.) : Frontiers in Propulsion Science, American Institute of Aeronautics and Astronautics 2009, and Davis, E.W., Chaps. 4 and 15. 
${ }^{4}$ Loll, R. : The Emergence of Spacetime or Quantum Gravity on Your Desktop, arXiv:0711.0273v2, 16 April 2008.

${ }^{5}$ Ambjorn,J., Jurkiewicz, J., R. Loll: The Self Organizing Quantum, Scientific American, August 2008.

${ }^{6}$ Ambjorn,J., Jurkiewicz, J., R. Loll: Quantum Gravity: the art of building spacetime, Chap. 18 in Quantum Gravity, ed. D. Oriti, Cambridge Univ. Press, 2009.

${ }^{7}$ Sarkar, U.: Particle and Astroparticle Physics, Taylor\&Francis 2008.

${ }^{8}$ Hasinger, G.: Das Schicksal des Universums, Goldmann 2009.

${ }^{9}$ Dröscher, W., J. Hauser: Gravity-Like Fields and Space Propulsion Concepts, AIAA 2008-5124, 44th AIAA/ASME/SAE/ASE, Joint Propulsion Conference \& Exhibit, Hartford, CT, 20-23 July 2008, 19 pp.

${ }^{10}$ Hobson, M.P., G. Efstathiou, A.N. Lasenby .: General Relativity, Cambridge Univ. Press, 2006.

${ }^{11}$ Smolin, L.: Atoms of Space and Time, Scientific American, January 2004.

${ }^{12}$ Dröscher,W., J. Hauser: Current Research in Gravito-Magnetic Space Propulsion, Paper O-42, 7th International Symposium on Launcher Technologies, 2-5 April 2007, Barcelona, Spain, 16 pp.

${ }^{13}$ Dröscher,W., J. Hauser: Advanced Propulsion Systems from Artificial Gravitational Fields, AIAA 2007-5595, 43th AIAA/ASME/SAE/ASE, Joint Propulsion Conference \& Exhibit, Cincinnati, OH, 8-11 July 2007, 15 pp.

${ }^{14}$ Dröscher,W., J. Hauser: Spacetime Physics and Advanced Propulsion Concepts, AIAA 2006-4608, 42nd AIAA/ASME/SAE/ASE, Joint Propulsion Conference \& Exhibit, Sacramento, CA, 9-12 July 2006, 20 pp., (available as revised extended version 20 August 2006 at www.hpcc-space.de).

${ }^{15}$ Stanford University: Gravity Probe B Final Report, einstein.stanford.edu, December 2008.

${ }^{16}$ Einstein, A.: On the Generalized Theory of Gravitation, Scientific American, April 1950, Vol 182, NO.4.

${ }^{17}$ Bruno, C., A.G. Accetura.: Advanced Propulsion Systems and Technologies, Today to 2020, AIAA 2008, 489 pp.

${ }^{18}$ Heim, B.: Vorschlag eines Weges einer einheitlichen Beschreibung der Elementarteilchen, Zeitschrift für Naturforschung, 32a, 1977, pp. 233-243.

${ }^{19}$ Kaku, M.: Quantum Field Theory, Oxford, 1993.

${ }^{20}$ Schmüser, P.: Feynman-Graphen und Eichtheorien fúr Experimentalphysiker [Lecture Notes in Physics), Springer 1998.

${ }^{21}$ Zee, A.: Quantum Field Theory in a Nutshell, Princeton University Press, 2003.

${ }^{22}$ Lahore, A., P., B. Pal: Quantum Field Theory, 2nd Ed., Alpha Science, 2004.

${ }^{23}$ Scheck, F.: The Higgs Mechanism and Spontaneous Symmetry Breaking, Chap. 11 in Noncommutative Geometry and the Standard Model of Elementary Particle Physics, eds. F. Scheck, H. Upmeier, W. Werner, Springer 2002.

${ }^{24}$ Tajmar, M. et al.: Hypothetical Gravity Control and Possible Influence on Space Propulsion, JournalofPropulsionandPower, Vol. 21, No. 4, July-August 2005.

${ }^{25}$ Heim, B. (ed. A. Resch): Mensch und Welt, Resch Verlag Innsbruck 2008.

${ }^{26}$ Heim. B., Dröscher, W.: Strukturen der Physikalischen Welt und ihrer nichtmateriellen Seite, Resch Verlag, Innsbruck, Austria, 1996, 2nd ed. 2007.

${ }^{27}$ Cardone, F. and R. Mignani: Energy and Geometry, World Scientific 2004.

${ }^{28}$ Woods C. et al.: Gravity Modification by High Temperature Superconductors, AIAA 2001-3363, 37 th AIAA/ASME /SAE/ASE, Joint Propulsion Conference \& Exhibit, Salt Lake City, Utah, 8-11 July 2001.

${ }^{29}$ Tajmar, M. et al.: Experimental Detection of the Gravitomagnetic London Moment, http://arxiv.org/abs/gr-qc/0603033, 2006.

${ }^{30}$ Tajmar, M. et al.: Measurement of Gravitomagnetic and Acceleration Fields Around Rotating Superconductors, STAIF AIP, February 2007.

${ }^{31}$ Tajmar, M. et al.: Search for Frame Dragging in the Vicinity of Spinning Superconductors, http://arxiv.org/abs/0707.3806v5, 14 September 2007, 14 pp. Note: This paper contains a comparison with the measurements by R.D. Graham et al. and also with the Stanford-NASA Gravity Probe B experiment.

${ }^{32}$ Graham, R. D. et al.: Experiment to Detect Frame Dragging in a Lead Superconductor, www2.phys.canterbury.ac.nz/ physrin/papers/SuperFrameDragging2007.pdf), 6 July 2007, 11 pp.

${ }^{33}$ Tajmar, M. et al.: Anomalous Fiber Optic Gyroscope Signals Observed Above Spinning Rings at Low Temperature, http://arxiv.org/abs/gr-qc/0603033, June 2008.

${ }^{34}$ Kiefer, C.: Quantum Gravity, Oxford University Press 2007.

${ }^{35}$ Ciufolini, I. et al.: Determination of frame-dragging using earth gravity models from CHAMP and GRACE, New Astronomy 11 (2006) 527-550.

${ }^{36}$ Veltmann, C.: Facts and Mysteries in Elementary Particle Physics, World Scientific 2003.

${ }^{37}$ Zwiebach, R.: Introduction to String Theory, Cambridge Univ. Press 2009, 2nd ed.

${ }^{38}$ Dröscher,W., J. Hauser: Guidelines For a Space Propulsion Device Based on Heim's Quantum Theory, AIAA 2004-3700, 40th AIAA/ASME/SAE/ASE, Joint Propulsion Conference \& Exhibit, Fort Lauderdale, FL, 11-14 July 2004, 31 pp.

${ }^{39}$ Dröscher,W., J. Hauser: Heim Quantum Theory for Space Propulsion Physics, AIP, STAIF, 2005, 10pp.

${ }^{40}$ Dröscher,W., J. Hauser: Magnet Experiment to Measuring Space Propulsion Heim-Lorentz Force, AIAA 2005-4321, 41st AIAA/ASME/SAE/ASE, Joint Propulsion Conference \& Exhibit, Tuscon, Arizona, 10-13 July 2005, 10 pp. 